\title{
FÓRMULAS DE MAGNITUD LOCAL PARA LA ZONA DEL PALETARÁ (CAUCA, COLOMBIA)
}

\author{
LOCAL MAGNITUDE SCALE FOR THE AREA OF PALETARÁ \\ (CAUCA, COLOMBIA)
}

\author{
Jaime Raigosa A. ${ }^{1}$, John Makario Londoño \\ *Email de correspondencia: jraigosa@sgc.gov.co
}

Recibido: 10/08/2017

Aceptado: 16/11/2017

Publicado en línea: 20/04/2018

Citación: Raigosa, J. y Londoño, J. (2018). Fórmulas de magnitud local para la zona del Paletará (Cauca, Colombia). Boletín Geológico, 44, 89-99.

\section{RESU MEN}

Se definió la primera fórmula de magnitud local para la zona del Paletará (ZP) a partir de una inversión de amplitudes de componentes horizontales de estaciones de banda ancha y corto periodo, simuladas en un sismograma Wood-Anderson, para sismos VT y tectónicos locales con profundidades menores a $37 \mathrm{~km}$ y distancias hipocentrales menores a $220 \mathrm{~km}$. Se invirtieron 628 sismos, con un total de 2848 amplitudes, en dieciocho estaciones sísmicas. La función de corrección por distancia encontrada para la ZP fue

$$
-\log \left(A_{\mathrm{o}}\right)=1,3541 \log (r / 17)+0,001639(r-17)+2
$$

donde $\log \left(A_{o}\right)$ es la corrección por distancia y $r$ es la distancia hipocentral. La fórmula de magnitud para la ZP queda definida así:

$$
M_{L}=\log \left(A_{m m}\right)+1,3541 \log (r / 17)+0,001639(r-17) 2+S,
$$

o alternativamente, según la formulación Iaspei:

$$
M_{L}=\log \left(A_{n m}\right)+1,3541 \log (r)+0,001639 r-2,37+S
$$

Donde $A_{m m}$ es la amplitud pico en milímetros simulada en un sensor Wood-Anderson, $A_{n m}$ es la amplitud pico en nanómetros simulada en un sensor Wood-Anderson, con una amplificación estática de 1, y $S$ es la corrección por estación debida a la amplificación de cada sitio.

Palabras clave: magnitud local, sismógrafo Wood-Anderson, atenuación.

Geólogo. Servicio Geológico Colombiano

2 Ph. D. en Geofísica. Servicio Geológico Colombiano 


\section{A B S T R A C T}

The determination of the local magnitude scale (ML) for the Paletará zone (PZ), Colombia was performed for the first time by the inversion of the horizontal components of 2848 amplitudes at 18 seismic stations for VT and local tectonic earthquakes with focal depths less than $37 \mathrm{~km}$ and focal distances less than $220 \mathrm{~km}$. The distance corrected function found in this study is

$$
-\log \left(A_{\mathrm{o}}\right)=1,3541 \log (r / 17)+0,001639(r-17)+2,
$$

where $-\log \left(A_{0}\right)$ is the empirically determined distance correction and $\mathrm{r}$ is the hypocentral distance. Accordingly, the local magnitude scale for $\mathrm{PZ}$ is defined as:

$$
M_{L}=\log \left(A_{m m}\right)+1,3541 \log (r / 17)+0,001639(r-17) 2+S,
$$

or according to IASPEI formulation:

$$
M_{L}=\log \left(A_{n m}\right)+1,3541 \log (r)+0,001639 r-2,37+S
$$

where $A_{m m}$ is the peak amplitude in mm simulated in a Wood-Anderson seismogram, $A_{n m}$ is the peak amplitude in nm simulated in a Wood-Anderson seismogram, with a magnification of 1 , and $S$ is the station correction due to differences in site amplification.

Keywords: local magnitude, Wood-Anderson seismograph, attenuation.

\section{INTRODUCCIÓN}

a magnitud local $\left(M_{L}\right)$ de un sismo, como fue definida originalmente por Richter (1935), es el logaritmo en base 10 de la amplitud cero-pico, medida en milímetros, registrada en un sismógrafo Wood-Anderson, de tal forma que un sismo de magnitud 3 registrado a 100 kilómetros de distancia tendrá una amplitud de $1 \mathrm{~mm}$, o lo que es equivalente, un sismo de magnitud cero tendrá una amplitud de $0,001 \mathrm{~mm}$ a esa misma distancia. Con el fin de tener comparaciones más significativas en regiones con atenuaciones muy diferentes, Hutton y Boore (1987) propusieron redefinir la escala de $M_{L}$ de tal forma que un sismo de magnitud 3 tenga una amplitud de $10 \mathrm{~mm}$ a $17 \mathrm{~km}$ de distancia, lo cual es consistente con la definición original de Richter. La determinación de $M_{L}$ es hoy en día una tarea rutinaria en cualquier observatorio sismológico o vulcanológico, y se utiliza para la estimación tanto del tamaño de un sismo como de la energía liberada por el mismo. Desde que Richter introdujo la fórmula de magnitud local (1935) para el sur de California, se han realizado numerosos trabajos en diferentes zonas para determinar las fórmulas de magnitud local, utilizando diferentes tipos de instrumentos, como sismómetros Wood-Anderson (Hutton y Boore, 1987), sismómetros de corto periodo (Greenhalgh y Singh, 1986; Baumbach et al., 2005), acelerómetros (Wu et al., 2005), sismómetros de banda ancha (Uhrhammer et al., 1996 Keir et al., 2006), o combinaciones de estos tipos de instrumentos (Bragato y Tento, 2005; Miao y Langston, 2007; Bobbio et al., 2009).

La correcta estimación de la magnitud local puede verse afectada por variaciones locales en la amplitud debido a la ubicación de la estación con respecto al patrón de radiación de la fuente, ruido local o estructura de la corteza y, en particular, a efectos de sitio (Havskov y Ottemöller, 2010), razones por las que se requiere ajustar el efecto de cada uno de ellos en la fórmula de magnitud para cada región. Por eso es importante determinar la fórmula de magnitud local para cada región en particular en lugar de usar fórmulas generales, como se viene haciendo en la actualidad, ya que estas pueden dar un estimativo erróneo de la magnitud: aumentándola o disminuyéndola, dependiendo principalmente de si el medio es menos o más atenuado que el del modelo asumido, y de 
las condiciones geológicas locales (efecto de sitio) de las estaciones sísmicas.

En Colombia se han realizado pocos estudios para determinar la fórmula de magnitud local, bien sea a nivel nacional o regional (Zollweg, 1990; Rengifo y Ojeda, 2004; Tamayo, 2011; Londoño, 2016).

La zona del Paletará (ZP) está ubicada en el centro-sur de Colombia, sobre el eje de la cordillera Central de Colombia, y separa la cadena volcánica de los Coconucos y el complejo volcánico Sotará. Varias de estas estructuras volcánicas, entre las que se destacan los volcanes Puracé y Sotará, presentan actividad sísmica y son monitoreadas permanentemente por el SGC.

En este trabajo se define por primera vez una fórmula de magnitud local para la ZP mediante la inversión de amplitudes pico de ondas sísmicas, leídas en sismogramas Wood-Anderson sintéticos, obtenidas a partir de registros de once estaciones sismológicas de banda ancha y siete de corto periodo operadas rutinariamente por el Observatorio Vulcanológico y Sismológico de Popayán (Ovspop) para el monitoreo de los volcanes activos Nevado del Huila, Puracé y Sotará, con el fin de mejorar la determinación de este parámetro para esta región, y para ser usado en la rutina diaria del Servicio Geológico Colombiano.

\section{MÉtodos, datos y PROCESAMIENTO}

El método utilizado en este estudio se basa en el trabajo de Nguyen et al. (2011), que fue adaptado por Londoño (2016) para la inversión de amplitudes en áreas pequeñas. La determinación de la magnitud local se basa en la máxima amplitud registrada (cero a pico) en un sismógrafo de torsión Wood-Anderson (WA), según la definición clásica de magnitud local. La relación entre el tamaño relativo del sismo y la amplitud según Richter (1935, 1958) está dada por

$M_{L}=\log A(\Delta)-\log A_{o}(\Delta)+S$,

donde $\log A$ es el logaritmo en base 10 de la amplitud cero a pico en mm en un sismograma WA; $\Delta$ es la distancia epicentral en $\mathrm{km}$; $-\log A_{o}$, es una corrección por distancia, y $S$ es la corrección empírica por estación. La corrección por distancia, $-\log A_{o}(\Delta)$, fue determinada por Hutton $y$ Boore (1987) de la siguiente forma: $-\log A_{o}=a \log (r / 100)+b \log (r-100)+3$,

donde $a$ y $b$ son los coeficientes empíricos de dispersión geométrica y atenuación anelástica, respectivamente; $r$ es la distancia hipocentral en $\mathrm{km}$, y el valor constante de 3 está dado por la definición original de Richter de $M_{L}$. La fórmula generalizada de magnitud local se define entonces así:

$M_{L}=\log (A)+a \log (r / 100)+b \log (r-100)+3$,

donde $A$ es la máxima amplitud (cero a pico) en mm en la componente vertical o en las componentes horizontales simuladas en un sismograma Wood-Anderson, y $r$ la distancia hipocentral en $\mathrm{km}$.

Cuando las variaciones en la atenuación dentro de los primeros 100 kilómetros son significativas, se puede ajustar la fórmula de Richter para determinar la magnitud en un área determinada con el fin de disminuir el efecto regional en la propagación de las ondas. Esto se hace mediante la normalización a $17 \mathrm{~km}$ en lugar de $100 \mathrm{~km}$, como originalmente se definió la fórmula, ya que cerca de la fuente sísmica se disminuye el efecto de las diferencias en las propiedades elásticas del medio y, por lo tanto, se podría esperar que las amplitudes de un sismo de una determinada magnitud sean similares independientemente de la región. Para ser consistente con la definición original, esto equivale a tener una amplitud de $10 \mathrm{~mm}$ en un sismograma simulado de un sismómetro de torsión Wood-Anderson para una magnitud de 3 (Hutton y Boore, 1987), o $1 \mathrm{~mm}$ (480 nm con magnificación 1) para una magnitud de 2. Utilizando esta definición, la fórmula de $M_{L}$ queda expresada así:

$M_{L}=\log (A)+a \log (r / 17)+b \log (r-17)+2+\mathrm{S}$

Iaspei ha propuesto una forma de definir la magnitud local:

$M_{L}=\log (A)+a \log (r)+b r+c$

El valor $c$ (magnitud de ajuste) se obtiene una vez se conocen los valores de $a$ y $b$, usando esta expresión:

$c=M_{L_{r e f}}-\log \left(A_{\text {ref }}\right)-\operatorname{alog}\left(r_{\text {ref }}\right)-b r_{\text {ref }}$ 
donde $M_{L_{r e f}}, A_{r e f} \mathrm{y} r_{r e f}$ se refieren a la magnitud, amplitud y distancia de referencia, respectivamente. Para una distancia $r_{r e f}$ de $100 \mathrm{~km}$ se tiene una $M_{L_{r e f}}=3$, y $A_{\text {ref }}=480 \mathrm{~nm}$. Para una distancia $r_{\text {ref }}$ de $17 \mathrm{~km}$ se tiene $M_{L_{r e f}}=2, \mathrm{y}$ $A_{\text {ref }}=480 \mathrm{~nm}$ (Havskov y Ottemöller, 2010). Siguiendo la metodología de Nguyen et al. (2011) para establecer una fórmula de magnitud local mediante la inversión de amplitudes simuladas en registro Wood-Anderson para zonas pequeñas, se hace una combinación de las ecuaciones (1) y (2), y se establece una corrección por distancia así:

$\sum_{k=1}^{m} M_{k} \delta_{i k}-\sum_{l=1}^{n} s_{l} \delta_{l j}-\operatorname{alog}\left(r_{i j} / 17\right)-b\left(r_{i j}-17\right)=$ $\log A_{i j}+2 i, k=1,2 \ldots m ; j, 1=1,2 \ldots n$,

donde $A_{i j}$ es la máxima amplitud horizontal cero a pico del $i$-ésimo evento registrado en la j-ésima estación; $M_{k}$ es la magnitud del $k$-ésimo evento; $s_{j}$ es la corrección de la $j$-ésima estación; $r_{i j}$ es la distancia hipocentral entre el $i$-ésimo evento, y la $j$-ésima estación; $\delta$ es la función "delta de Kronecker"; $m$ es el número de eventos, y $n$ es el número de estaciones. $a, b, M_{k}$ y $S_{l}$ son parámetros por determinar y representan la dispersión geométrica, atenuación inelástica, magnitud y corrección de la estación, respectivamente. La ecuación (7) se puede escribir en forma matricial, así:

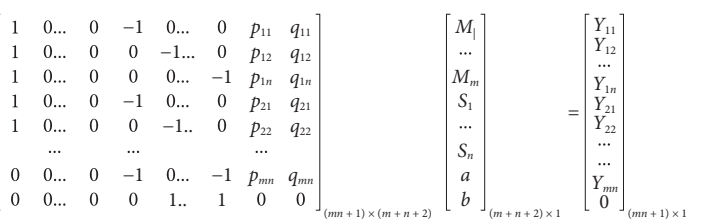

o $\boldsymbol{G} \boldsymbol{u}=d$, que es un sistema de $(m \times n)+1$ de ecuaciones linealmente independientes, con $m+n+2$ parámetros por determinar. De la ecuación (8) se tiene

$p_{i j}=-\log \left(r_{i j} / 17\right) ; q_{i j}=-\left(r_{i j}-17\right)$;
El vector $\boldsymbol{u}$ se puede encontrar mediante la matriz inversa generalizada de $\boldsymbol{G}\left(\boldsymbol{G}^{-g}\right)$ usando la técnica de descomposición de valores únicos propuesta por Miao y Langston (2007), quienes adoptan una inversión lineal de un paso sin iteración para un problema de inversión sobredeterminado, como el de la ecuación (8). La última línea de esta expresión matricial muestra que el promedio de la corrección por estaciones es igual a cero (i.e. $\sum_{l=1}^{n} S_{l}=0$; Hutton y Boore, 1987). Losvalores delos coeficientes $a$ (dispersión geométrica) y $b$ (atenuación anelástica) obtenidos en la inversión determinan la forma de la curva $-\log A_{o}$, de acuerdo con la ecuación (2). Una vez conocidos $a$ y $b$, se puede obtener el valor $c$ mediante la ecuación (6).

Las amplitudes que se obtuvieron en un sismograma Wood-Anderson se simularon usando la amplificación estándar del instrumento (período natural de 0,8 segundos, damping de 0,8 y amplificación estática de 2080) en las estaciones de banda ancha y corto periodo seleccionadas una vez corregidas por respuesta instrumental y convertidas a desplazamiento. Estos valores, en el instrumento Wood-Anderson, son apropiados para el rango de frecuencias usados en este trabajo (Bormann, 2013).

Se seleccionaron 628 sismos volcano-tectónicos y tectónicos locales ocurridos en la $\mathrm{ZP}$, registrados en once estaciones de banda ancha y siete de corto periodo, con buena distribución azimutal, con el fin de cubrir toda la zona y así obtener una mejor curva de atenuación, con magnitudes coda (Mc) en el rango de 1,3 y 4,5, con profundidades de hasta $37 \mathrm{~km}$ y distancias epicentrales de hasta $220 \mathrm{~km}$, tomando como centro la zona media de la ZP. La figura 1 muestra los sismos y las estaciones usados en este trabajo, y la figura 2, la distribución por rangos de distancia epicentral de la muestra. Para la inversión se usaron un total de 2848 amplitudes en cada componente (dos horizontales y una vertical), que se obtuvieron removiendo la respuesta instrumental de cada uno de los registros digitales de las estaciones, con el fin de obtener las trazas en $\mathrm{nm} / \mathrm{seg}$. Estas amplitudes luego se convirtieron a mm en registros WoodAnderson simulados, y se extrajeron las amplitudes máximas cero a pico de cada componente. Para obtener la fórmula de magnitud local se utilizó el promedio de la máxima amplitud en las dos componentes horizontales. 


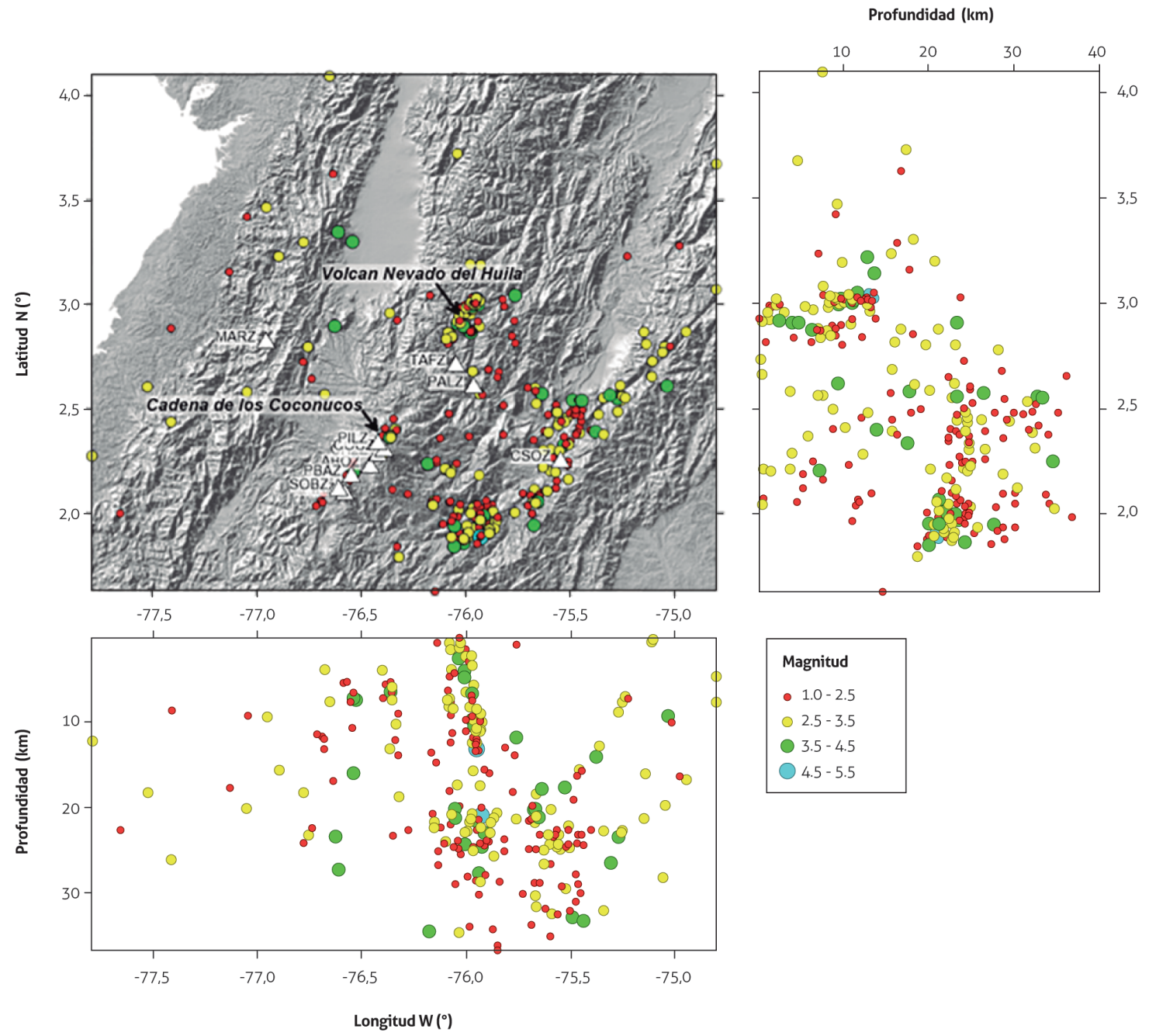

Figura 1. Localización hipocentral de los sismos (círculos de colores) y estaciones (triángulos blancos) usados para la determinación de las

fórmulas de magnitud local para la ZP. Los tamaños de los círculos representan la magnitud local calculada con la ecuación para California Fuente: autores

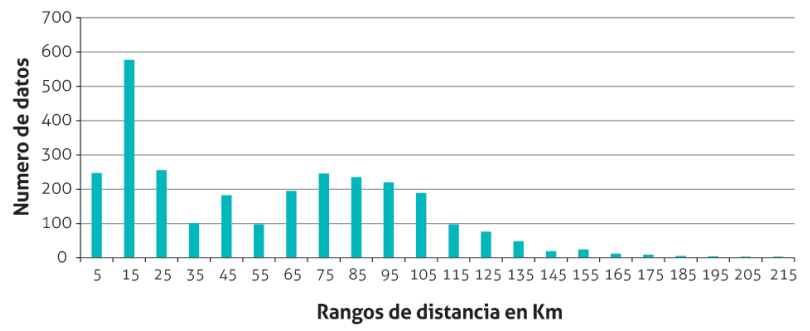

Figura 2. Número de sismos por rangos de distancia epicentral de la muestra utilizada; se observa que por encima de $140 \mathrm{~km}$ el número de sismos es muy bajo

Fuente: autores

\section{Resultados}

A partir de los resultados de la inversión de las amplitudes se obtuvieron los valores de $a$ y $b$, que definieron la curva de atenuación local para la ZP así:

$-\log \left(A_{o}\right)=1,3541 \times \log (r / 17)+0,001639 \times(r-17)+2,(10)$

donde $\log \left(A_{o}\right)$ es la corrección por distancia, y $r$ es la distancia hipocentral. Por lo tanto, la fórmula generalizada de magnitud local para la ZP se expresa así:

$M_{L}=\log (A)+1,3541 \times \log (r / 17)+0,001639 \times(r-17)+2,(11)$ 
donde $A$ es la amplitud pico en mm simulada en un sismograma Wood-Anderson.

En el proceso de inversión, los valores $a$ y $b$ se obtienen a partir de las amplitudes leídas en los sismogramas, $\mathrm{y}$, por lo tanto son independientes de los valores $M_{\text {Lref }} \mathrm{y} r_{\text {ref }}$ utilizados para determinar la escala de magnitud, razón por la cual la escogencia de estos valores no altera la curva de atenuación $-\log \left(A_{o}\right)$ (figura 3). La figura 4 muestra la curva de atenuación local obtenida para la ZP. En esta figura se observa un buen ajuste con respecto a los datos observados, con una desviación estándar de 0,206.

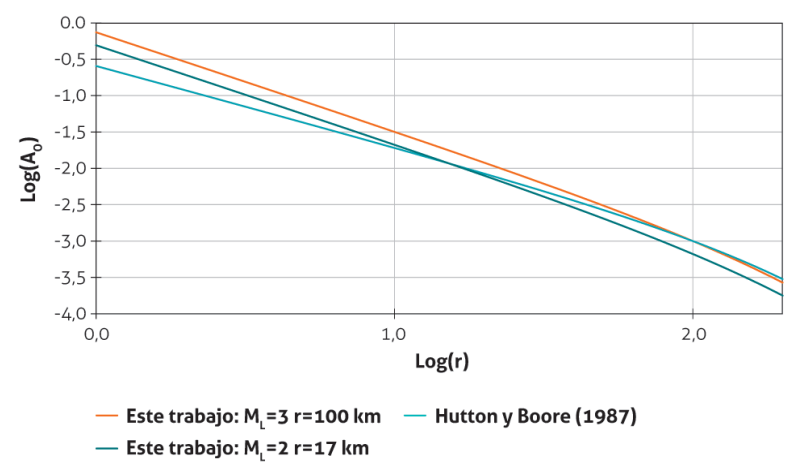

Figura 3. Curva de $\log \left(A_{\mathrm{o}}\right)$. En color azul, la curva obtenida por Hutton y Boore (1987) utilizando la definición original de Richter, según la cual un sismo de magnitud 3 a 100 kilómetros de distancia registra una amplitud de $1 \mathrm{~mm}$. En colores negro y amarillo, las curvas obtenidas en este trabajo. Obsérvese el desplazamiento de la curva, hacia arriba o hacia abajo, dependiendo del punto de anclaje (100 km para la curva amarilla y $17 \mathrm{~km}$ para la negra), pero conservando la forma

Fuente: autores

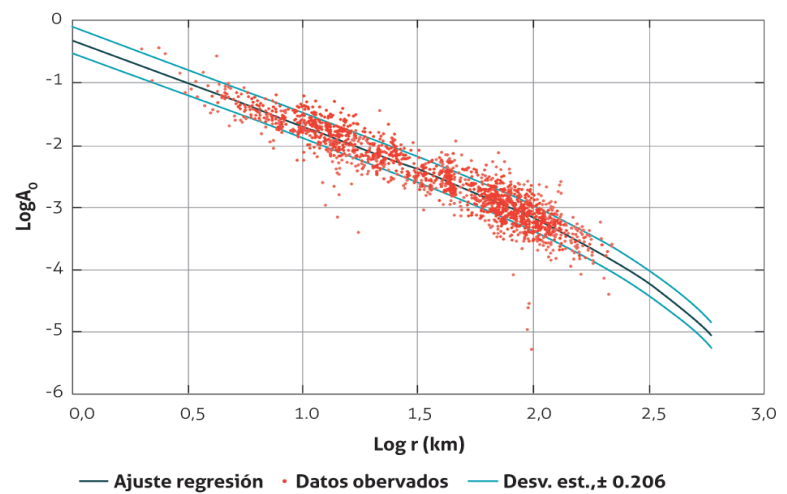

Figura 4. Comparación entre la función de corrección por distancia calculada $\left(\log A_{o}\right)$ y los valores de amplitud obtenidos de sismogramas simulados Wood-Anderson utilizados en este estudio usando el promedio de las componentes NS y EW para la ZP

Fuente: autores
La figura 5 muestra la corrección por distancia en escala logarítmica para la ZP y su comparación con el trabajo original de Richter para la zona de California.

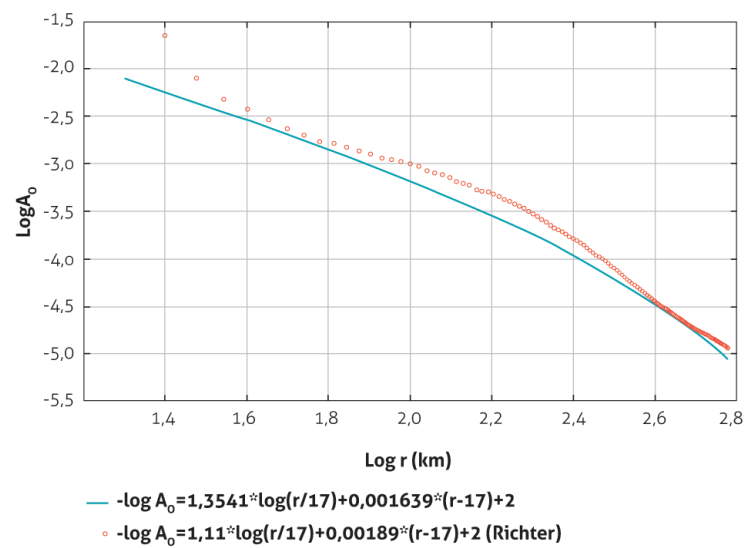

Figura 5. Curva de atenuación para la ZP. La curva azul corresponde a la corrección por distancia empírica; los círculos rojos representan la curva para California (Richter, 1935, 1958) Fuente: autores

Alternativamente, la fórmula de magnitud local para la ZP puede expresarse, de acuerdo con el formato Iaspei, así:

$M_{L}=\log (A)+1,3541 \times \log (r)+0,001639 \times r-2,37+S$,

donde $A$ es la amplitud pico en nm simulada en un sismograma Wood Anderson, y $S$ es la corrección por estación. La constante (2,37, nivel base) se obtiene al realizar una regresión para la relación $\log (A)+b \times r v s \log (r)$ y aplicar la ecuación (6) (Havskov y Ottemöller, 2010). El valor de la magnitud final resulta luego de calcular la magnitud en varias estaciones y calcular el promedio. Como se puede observar en (8), el proceso de inversión incluye la determinación de la corrección por efecto de sitio $(S)$ en unidades de magnitud, con la restricción de que la sumatoria de las correcciones en todas las estaciones sea igual a cero. La tabla 1 muestra los resultados. 
Tabla 1. Corrección de la magnitud para cada estación utilizada y tipo de roca en la que está instalada

\begin{tabular}{|c|c|c|c|}
\hline Estación & Tipo de estación & Corrección (unidades de magnitud) & Tipo de roca \\
\hline BUC & $\mathrm{CP}$ & $-0,702$ & DV \\
\hline TAF & $\mathrm{BA}$ & $-0,604$ & SR \\
\hline PAL & BA & $-0,452$ & SR \\
\hline OS2 & $\mathrm{CP}$ & $-0,262$ & LA \\
\hline MAR & BA & $-0,184$ & DF \\
\hline PIR & BA & $-0,097$ & LA \\
\hline $\mathrm{COC}$ & $\mathrm{BA}$ & $-0,054$ & LA \\
\hline SHA & $\mathrm{CP}$ & 0,003 & LA \\
\hline $\mathrm{CO} 2$ & $\mathrm{CP}$ & 0,048 & LA \\
\hline SOB & BA & 0,090 & SR \\
\hline CSO & $\mathrm{BA}$ & 0,105 & LA \\
\hline $\mathrm{ABO}$ & BA & 0,125 & LA \\
\hline SOT & $\mathrm{CP}$ & 0,136 & LA \\
\hline $\mathrm{CH} 2$ & $\mathrm{BA}$ & 0,139 & LA \\
\hline VR2 & $\mathrm{CP}$ & 0,177 & LA \\
\hline PIL & BA & 0,391 & LA \\
\hline LAR & $\mathrm{CP}$ & 0,487 & LA \\
\hline PBA & $\mathrm{BA}$ & 0,655 & $\mathrm{LA}$ \\
\hline
\end{tabular}

$\mathrm{BA}=$ banda ancha, $\mathrm{CP}=$ corto período, $\mathrm{LA}=$ lava andesítica, $\mathrm{SR}=$ suelo residual, $\mathrm{DV}=$ depósitos volcánicos, $\mathrm{DF}=$ depósitos fluviales Fuente: autores

\section{AnÁLISIS}

La figura 3 muestra que la curva de atenuación encontrada para la ZP indica una mayor atenuación que la curva encontrada para California por Hutton y Boore (1987). Dado que la curva encontrada en este estudio fue anclada a $17 \mathrm{~km}$ de distancia, esta predice valores menores de magnitud a distancias menores de $17 \mathrm{~km}$ y valores mayores a distancias mayores de $17 \mathrm{~km}$. La figura 6 muestra la relación entre la $M_{L}$ encontrada en este trabajo y la $M_{L}$ calculada rutinariamente en el Ovspop ( $M_{L}$ actual): en ella se observa que podría haber una subestimación considerable de la magnitud local en el cálculo rutinario en el Ovspop del Servicio Geológico Colombiano (SGC), hasta de una unidad de magnitud o más, además de que la comparación de ambos valores muestra una gran dispersión. Como explicación de esto podría mencionarse que la utilización de los valores definidos para California en los cálculos actuales, que predicen un modelo de corteza menos atenuada que la encontrada en el presente estudio, implican que un valor de amplitud registrado esté asociado a un valor menor de magnitud, y, por lo tanto, una subestimación del valor real. Respecto a la dispersión de los datos, la no utilización de los valores de corrección para las estaciones hace que las magnitudes calculadas dependan no solamente de la fórmula, sino también, en gran medida, de las estaciones utilizadas, pues, como se mostró en la tabla 1 , hay estaciones, como BUC, TAF, LAR y PBA, que requieren de correcciones notables.

Dado que tanto el Ovspop como la RSNC rutinariamente deben calcular y reportar información de sismos ocurridos en la ZP, se realizó también una comparación de las $M_{L}$ obtenidas con la fórmula definida en este trabajo, con las $M_{L}$ reportadas por la Red Sismológica Nacional de Colombia (RSNC), que utiliza la fórmula calculada por Rengifo y Ojeda (2004), $-\log \left(A_{o}\right)=1,019 \times \log (r / 100)+0,0016 \times(r-100)+3$, que es muy similar a la encontrada para California. La figura 7 muestra esta comparación, y en ella se observa de manera clara que los valores reportados por la RSNC son menores que los encontrados en este trabajo, aunque la dispersión es menor y la curva de ajuste mejor que las encontradas en la comparación con los datos del Ovspop. 


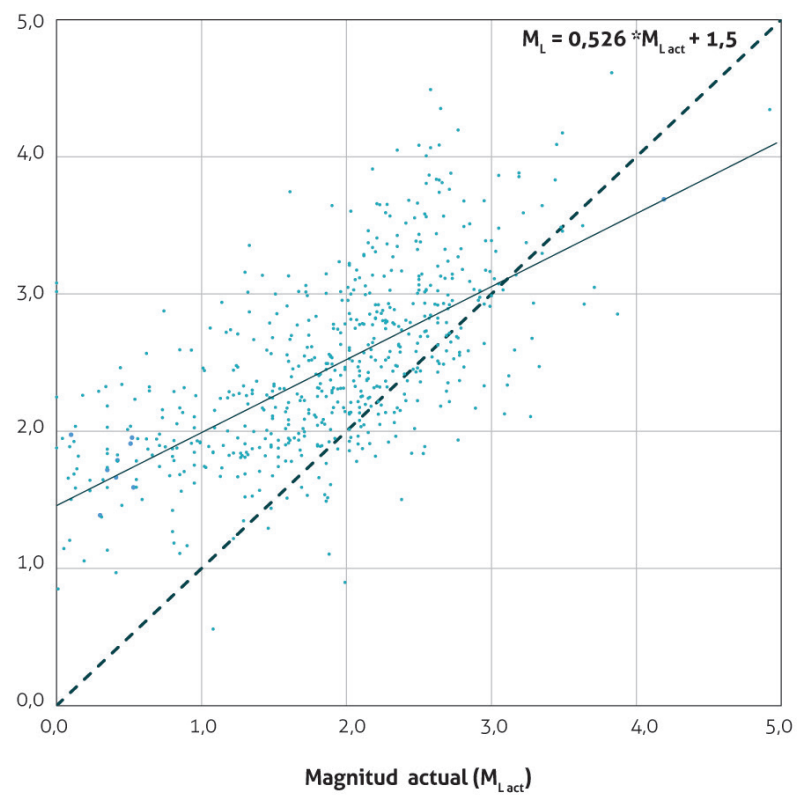

Figura 6. Relación entre la $M_{L}$ encontrada en este trabajo y la $M_{L}$ calculada rutinariamente en el Ovspop (Magnitud actual, $M_{L_{a c t}}$ ), para sismos VT y tectónicos locales registrados por la red de monitoreo del Ovspop. La línea continua representa la curva de ajuste. Como referencia se grafica la relación $M_{L}=M_{L_{a c t}}$ (línea punteada) Fuente: autores

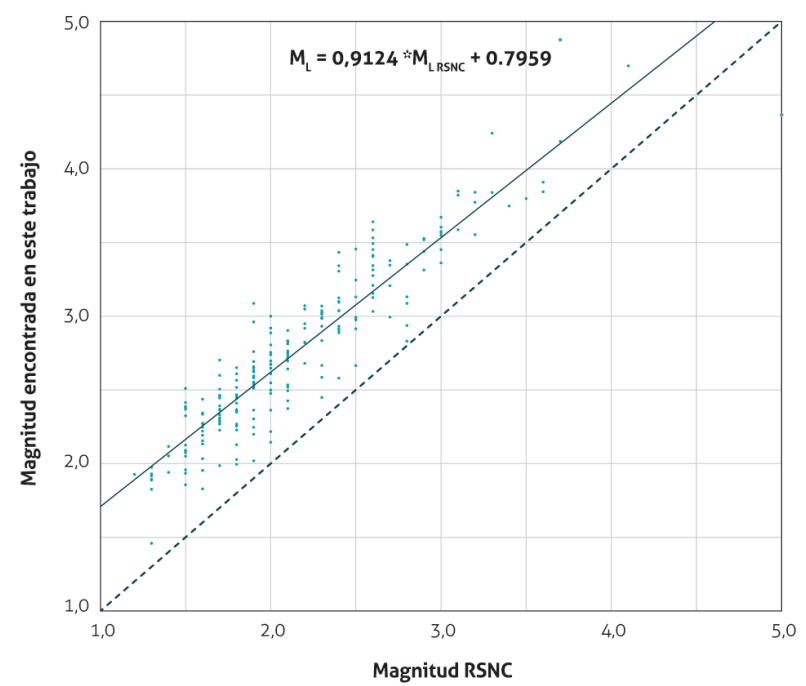

Figura 7. Relación entre la $M_{L}$ encontrada en este trabajo y la $M_{L}$ calculada rutinariamente en la RSNC $\left(M_{L_{R S N C}}\right)$ para sismos VT y tectónicos locales registrados por la red de monitoreo del Observatorio. La línea continua representa la curva de ajuste. Como referencia se grafica la relación $M_{L}=M_{L}$ (RSNC) (línea punteada)

Fuente: autores
La definición de una fórmula de magnitud local para la ZP permite homogeneizar los diferentes valores de magnitud que se tienen actualmente. Hasta la fecha, una parte de las magnitudes ha sido calculada con la fórmula de magnitud coda (1993-2010), otra parte se ha calculado con magnitud de coda y con $M_{L}$ usando la fórmula de San Francisco (2010-2017), así que, en las bases de datos, los sismos tienen asignadas ambas magnitudes; debido a esto, ha sido fácil observar las grandes diferencias que existen entre ambos valores. Una causa identificada es la subjetividad que existe en la asignación de la coda de un sismo, y, por lo tanto, de la magnitud asociada, ya que esto depende básicamente del criterio de quien asigna dicho valor. Esta situación hace que el cálculo de la energía sísmica no sea homogéneo, lo que podría, en algunos casos, hacer que se subvalore la energía, y, en otros, que se sobrevalore. Asimismo, realizar estudios sísmicos en los que la magnitud sea un parámetro de selección o de análisis para la distribución espacio-temporal puede conducir a interpretaciones erróneas de los resultados mientras no se estandarice la forma en que se estima la magnitud de un sismo.

Adicionalmente, los resultados obtenidos (en las estaciones de banda ancha y corto período) se compararon con los resultados obtenidos utilizando solo las estaciones de banda ancha. La figura 8 muestra las curvas de corrección por distancia y las diferencias promedio, para intervalos de $10 \mathrm{~km}$, entre las amplitudes calculadas usando la corrección por distancia obtenida (ecuación 12) y las amplitudes observadas. Aunque la utilización de ambos tipos de estaciones muestra una curva más atenuada, se puede apreciar que las diferencias son pequeñas, tanto en los promedios de amplitud como en los errores estándar. La figura 9 compara los valores de magnitud utilizando ambos conjuntos de estaciones; en ella se observa que dichos valores son muy similares y que la dispersión es baja, lo que permite concluir que es válida la utilización de estaciones de corto periodo para el cálculo de $M_{L}$, y que es posible utilizar estaciones de corto periodo en el cálculo rutinario de $M_{L}$ (Bormann, 2013).

Los valores obtenidos de correcciones de magnitud por estación reflejan en gran medida el efecto de sitio (Miao y Langston, 2007). Así, estaciones con valores negativos tendrán una amplificación de la amplitud debido a que puede tratarse de un suelo poroso, poco consolidado o de rocas 


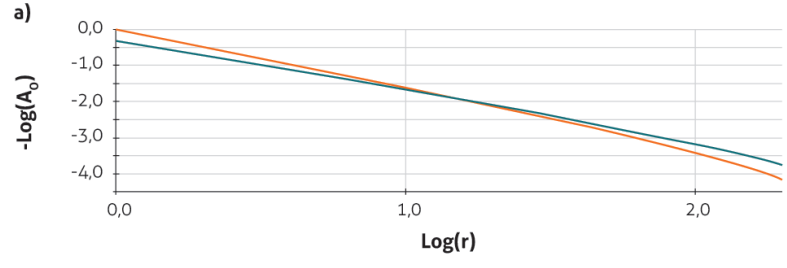

$-a=1,5695, b=0,002506, c=2,37-\mathrm{BA}+\mathrm{CP} a=1,3541, b=0,001639, c=2,65$

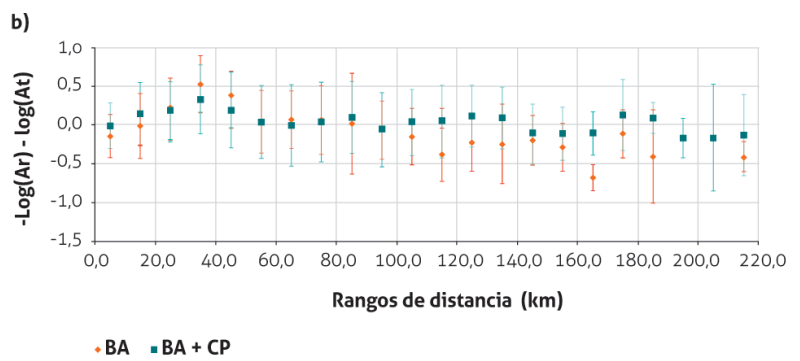

Figura 8. Comparación de resultados obtenidos usando solo estaciones de banda ancha (BA) y banda ancha y corto periodo combinadas $(\mathrm{BA}+\mathrm{CP})$. (a) Función de corrección por distancia Se presentan los valores de $a, b$ y $c$ de la fórmula de magnitud. (b) Diferencias promedio de valores de amplitud calculados versus observados, por rangos de distancia cada $10 \mathrm{~km}$. Las barras indican el error estándar

Fuente: autores

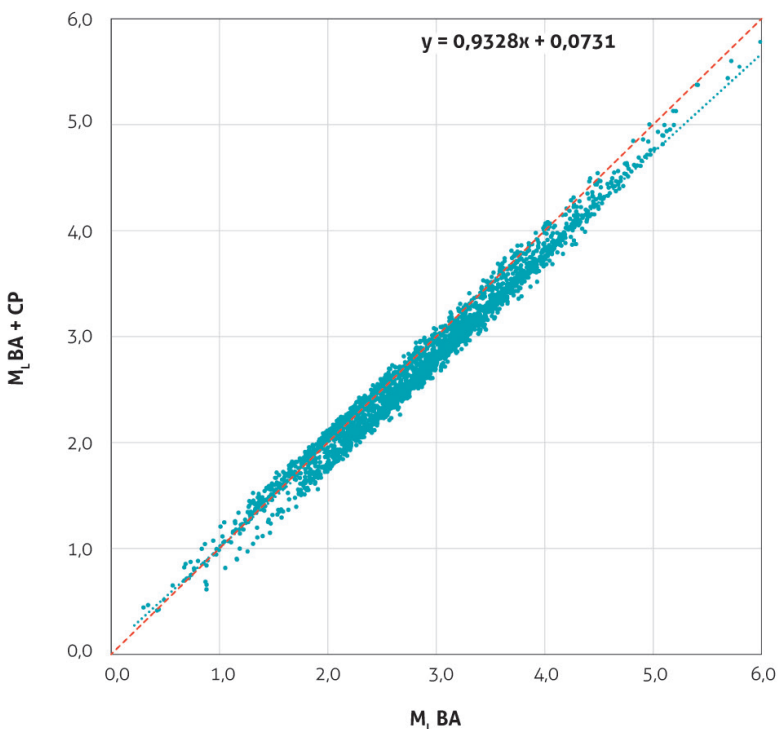

Figura 9. Comparación de magnitudes calculadas utilizando las funciones de corrección por distancia obtenidas solo con estaciones de banda ancha y con estaciones de banda ancha (BA) y corto periodo (CP) combinadas. La línea roja punteada representa la relación $M_{L_{B A}}=M_{L_{B A}}+C P$. La curva azul punteada representa el ajuste

Fuente: autore meteorizadas, y los valores positivos representarían rocas sólidas o competentes. Las estaciones BUC, PAL y TAF presentan correcciones negativas notables de la magnitud (amplificación), mientras que las estaciones PBA, LAR y PIL presentan correcciones positivas altas (atenuación). Estos valores pueden interpretarse como efectos de la geología local en las estaciones BUC, PAL y TAF, que están ubicadas sobre suelos blandos, mientras que PBA, LAR y PIL están ubicadas sobre lavas macizas (Marquínez et al., 2003). El resto de las estaciones presenta correcciones bajas o moderadas (tabla 1).

Finalmente, se realizó un recálculo de la magnitud local y energía sísmica de los sismos considerados en este trabajo usando la relación de la ecuación (11), y se compararon con la energía y magnitud coda usados en la rutina diaria y en los informes periódicos que se emiten en el Ovspop. Ambos cálculos se hicieron con la fórmula de energía sísmica de Gutenberg y Richter (1956). La figura 10 muestra los resultados. En esta figura resulta evidente que la energía sísmica es mucho mayor con la nueva fórmula de magnitud $\left(M_{L}\right)$ que con la magnitud coda $\left(M_{c}\right)$, pues llega a ser más de una unidad de magnitud superior con la nueva fórmula.

Estos resultados sugieren que la energía sísmica liberada en la zona del Paletará puede estar siendo subvalorada, lo que implicaría que se requiere una reevaluación de la energía sísmica liberada por algunos volcanes y fallas activas.

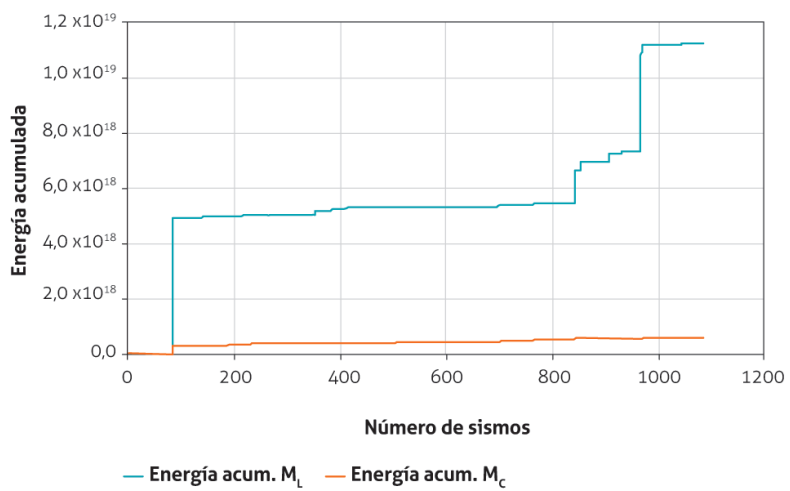

Figura 10. Energía sísmica acumulada diariamente, recalculada para los sismos considerados en este trabajo. La línea de color naranja corresponde a la energía calculada con la magnitud coda $\left(M_{c}\right)$, y la línea azul corresponde a la energía calculada con la nueva fórmula de magnitud

Fuente: autores 


\section{Conclusiones}

Se determinó la curva de atenuación local o corrección por distancia para la ZP usando un método de inversión de amplitudes, simulando un instrumento Wood-Anderson, y a partir de ella se obtuvo la fórmula de magnitud local. La curva encontrada es más atenuada que la curva definida para la zona de California utilizada actualmente para calcular las magnitudes en el Ovspop, lo cual sugiere una subestimación actual en la determinación de $M_{L}$. Asimismo, la magnitud coda presenta en algunos casos una subestimación de una unidad de magnitud, además de desviaciones sustanciales debido a la subjetividad en la lectura de la duración coda. Las correcciones encontradas para las estaciones, en unidades de magnitud, son consistentes con la geología local, y se presentan correcciones negativas grandes para estaciones ubicadas en suelos blandos (BUC, TAF, PAL) y correcciones positivas grandes para estaciones ubicadas en rocas duras (PBA, LAR, PIL). Existen otras estaciones ubicadas sobre roca firme que presentan valores de corrección cercanas a cero; al respecto se debe recordar que estas correcciones son relativas a la red utilizada y con un promedio de cero. De todas formas, es importante investigar por qué la estación PBA presenta una diferencia tan grande respecto a otras estaciones ubicadas sobre roca firme, como $\mathrm{CH} 2$ o VR2.

\section{Referencias Bibliográficas}

Baumbach, M., Bindi, D., Grosser, H., Milkereit, C., Paralai, S., Wang, R., et al. (2005). Calibration of an $M_{L}$ scale in Northwestern Turkey from 1999 Izmit aftershocks. Bulletin of the Seismological Society of America, 93(5), 2289-2295. Doi: 10.1785/0120020157.

Bobbio, A., Vassallo, M. y Festa, G. (2009). A local magnitude scale for Southern Italy. Bulletin of the Seismological Society of America, 99(4), 2461-2470. Doi: 10.1785/0120080364.

Bormann, P. (2013). Magnitude calibration formulas and tables, comments on their use and complementary data. New Manual of Seismological Observatory Practice 2 (NMSOP-2). Potsdam: Deutsches GeoForschungsZentrum. Doi: 10.2312/GFZ.NMSOP-2_DS_3.1.
Bragato, P. y Tento, A. (2005). Local magnitude in Northeastern Italy. Bulletin of the Seismological Society of America, 95(2), 576-591. Doi: 10.1785/0120040100.

Greenhalgh, S. y Singh, R. (1986). A revised magnitude scale for South Australian earthquakes. Bulletin of the Seismological Society of America, 76(3), 757-769.

Gutenberg, B. y Richter, C. (1956). Magnitude and energy of earthquakes. Annals of Geophysics, 9(1), 1-15. Doi: 10.4401/ag-5590.

Havskov, J. y Ottemöller, L. (2010). Routine data processing in earthquake Seismology. Dordrecht: Springer. Doi: 10.1007/978-90-481-8697-6.

Hutton, L. y Boore, D. (1987). The $M_{L}$ Scale in Southern California. Bulletin of the Seismological Society of America, 77(6), 2074-2094.

Keir, D., Stuart, W., Jackson, A. y Ayele, A. (2006). Local earthquake magnitude scale and seismicity rate for Ethiopian rift. Bulletin of the Seismological Society of America, 96(6), 2221-2230. Doi: 10.1785/0120060051.

Londoño, J. (2016). Definición de fórmulas de magnitud local para el área del complejo volcánico cerro Bravo-cerro Machín, Colombia. Bogotá: Servicio Geológico Colombiano.

Marquínez, G., Rodríguez, Y., Terraza, R. y Martínez, M. (2003). Geología de la plancha 365-Coconucos, escala 1:100.000. Mapa. Bogotá: Ingeominas.

Miao, Q. y Langston, C. (2007). Empirical distance attenuation and the local magnitude scale for the central US. Bulletin of the Seismological Society of America, 97(6), 2137-2151. Doi: 10.1785/0120060188.

Nguyen, L., Ting-Li, L., Yih-Min, W., Bor-Shouh, H., Chien-Hsin, C., et al. (2011). The first $\mathrm{M}_{\mathrm{L}}$ scale for north of Vietnam. Journal of Asian Earth Sciences, 40(1), 279-286. Doi: 10.1016/j.jseaes.2010.07.005.

Rengifo, F. y Ojeda, A. (2004). Inversión de amplitudes de registros sísmicos para el cálculo de magnitud $\mathrm{Ml}$ en Colombia. Memorias I Congreso Latinoamericano de Sismología. II Congreso, Colombiano de Sismología. Armenia.

Richter, C. (1935). An instrumental earthquake magnitude scale. Bulletin of the Seismological Society of America, 25(1), 1-32.

Richter, C. (1958). Elementary seismology. San Francisco: WH Freeman and Company. 
Tamayo, M. (2011). Determinación de la escala de magnitud local a partir de inversión de amplitudes y magnitud coda para sismos volcánicos del Nevado del Ruizy cerro Machín (tesis de pregrado). Universidad de Caldas, Manizales, Colombia.

Uhrhammer, R. Loper, S. y Romanowicz, B. (1996). Determination of local magnitude using BDSN broadband records. Bulletin of the Seismological Society of America, 86(5), 1314-1330.
Wu Y., Allen, R. y Wu, C. (2005) Revised $M_{L}$ determination for crustal earthquakes in Taiwan. Bulletin of the Seismological Society of America, 95(6), 2517-2524. Doi: 10.1785/0120050043.

Zollweg, J. (1990). Seismicity following the 1985 eruption of Nevado del Ruiz, Colombia. Journal of Volcanology and Geothermal Research, 41(1-4), 355-367. 